\section{From Mice to Humans: The Exocrine Pancreas Does Not Matter in Human GLP-1 Receptor Imaging}

TO THE EDITOR: Noninvasive determination of pancreatic $\beta$-cell mass (BCM) in humans is key to understanding the pathophysiology of type 1 diabetes (T1D) and type 2 diabetes. It appears that $\mathrm{BCM}$ and $\beta$-cell function are not directly linked and that $\beta$-cell dysfunction is a key pathophysiologic parameter. The main challenge of pancreatic $\beta$-cell imaging is to use a highly specific tracer molecule so that the signal originating from the pancreas reflects actual BCM (1).

Khera and coworkers show that reduction of exocrine pancreatic uptake by GLP-1 receptor (GLP-1R) blocking with fluorescent exendin improves in vivo imaging of pancreatic $\beta$-cells in mice (2). We have previously demonstrated that, as opposed to mice, rats do not display exocrine pancreatic uptake, as confirmed by quantitative polymerase chain reaction (3) and immunohistochemical GLP-1R staining (4). The human pancreas shows an even higher endocrine-to-exocrine ratio (3), and single-cell RNA sequencing demonstrates specificity of the GLP-1R for human $\beta$ - and $\delta$-cells (5).

Insulin-positive islets are present in pancreata of people with long-standing T1D, indicating that $\beta$-cell dysfunction plays an important role in T1D pathophysiology (6). Pancreatic exendin uptake in individuals with T1D therefore reflects dysfunctional BCM (representing highly important information about the pathophysiology of T1D) and not uptake in non- $\beta$-cells, explaining “ ... the lack of clinical distinction between healthy volunteers and subjects with long-term diabetes ...." as claimed by Khera et al. (2).

We have recently shown in people with long-standing T1D that exendin uptake in human pancreata can indeed drop to background levels, a strong argument against exocrine pancreas uptake. About half the tested individuals showed significant pancreatic uptake, pointing toward residual $\mathrm{BCM}$ (7), in line with the concept of $\beta$-cell dysfunction in T1D (6). Immunohistochemical analysis of human pancreata from individuals with long-standing T1D indeed confirms the presence of numerous GLP-1R-expressing $\beta$-cells and staining of $\delta$-cells, the latter explaining the low residual uptake after complete loss of $\beta$-cells. Data from healthy individuals confirm exclusive staining of $\beta$ - and $\delta$-cells and no staining in exocrine pancreas (7). These data are confirmed by the finding that ex vivo autoradiography of human pancreatic tissue shows only background uptake in the exocrine pancreas (comparable to rats) (8).

Finally, in view of the small differences in exocrine pancreatic uptake between wild-type and GLP-1R knockout mice, shown by Khera and coworkers (Fig. 1) (2), and the minor uptake reduction after blocking (2), the GLP-1R does not play an important role in exocrine pancreas uptake, as shown previously (4).

Therefore, although the approach presented by Khera and coworkers (2) is highly interesting, the practical value is limited to mouse imaging. In addition, high pharmacologic doses of (fluores-

\footnotetext{
(C) 2021 by the Society of Nuclear Medicine and Molecular Imaging.
}

cent) exendin may lead to receptor saturation phenomena disturbing the linear correlation between tracer uptake and $\mathrm{BCM}(1,3)$.

\section{REFERENCES}

1. Eriksson O, Laughlin M, Brom M, et al. In vivo imaging of beta cells with radiotracers: state of the art, prospects and recommendations for development and use. Diabetologia. 2016;59:1340-1349.

2. Khera E, Zhang L, Roberts S, et al. Blocking of glucagonlike peptide-1 receptors in the exocrine pancreas improves specificity for $\beta$-cells in a mouse model of type 1 diabetes. J Nucl Med. 2019;60:1635-1641.

3. Brom M, Woliner-van der Weg W, Joosten L, et al. Non-invasive quantification of the beta cell mass by SPECT with ${ }^{111}$ In-labelled exendin. Diabetologia. 2014;57: 950-959.

4. Willekens SMA, Joosten L, Boerman OC, et al. Strain differences determine the suitability of animal models for noninvasive in vivo beta cell mass determination with radiolabeled exendin. Mol Imaging Biol. 2016;18:705-714.

5. Segerstolpe Å, Palasantza A, Eliasson P, et al. Single-cell transcriptome profiling of human pancreatic islets in health and type 2 diabetes. Cell Metab. 2016;24:593-607.

6. Oram RA, Sims EK, Evans-Molina C. Beta cells in type 1 diabetes: mass and function; sleeping or dead? Diabetologia. 2019;62:567-577.

7. Boss M, Kusmartseva I, Woliner-van der Weg W, et al. In-exendin spect imaging suggests presence of residual beta cells in patients with longstanding type 1 diabetes [abstract]. Diabetologia. 2020;63:S22-S23.

8. Gotthardt M, Jansen TJP, Buitinga M, et al. Validation of exendin for beta cell imaging: ex vivo autoradiography of human pancreas demonstrates specific accumulation of radiolabeled exendin in islets of Langerhans [abstract]. Diabetologia. 2020;63:S179-S180.

\author{
Martin Gotthardt* \\ Lieke Joosten \\ Theodorus J.P. Jansen \\ Maarten Brom \\ Marti Boss \\ Stefanie M.A. Willekens \\ * Radboud University Medical Center \\ Nijmegen, The Netherlands \\ E-mail: martin.gotthardt@radboudumc.nl
}

Published online Feb. 19, 2021.

DOI: 10.2967/jnumed.120.259184

\section{Reply: From Mice to Humans: The Exocrine Pancreas Does Not Matter in Human GLP-1 Receptor Imaging}

REPLY: I want to thank Gotthardt and colleagues for bringing this important clinical question to the forefront of discussion - the independent measurement of $\beta$-cell mass and $\beta$-cell function (1). Indeed, this distinction has major implications for both type 1 and type 2 diabetes research. Their group and others have pioneered glucagon-like peptide-1 receptor (GLP-1R) imaging in the clinic, moving this research beyond preclinical animal models. Despite great progress, significant challenges remain. 


\section{CHALLENGES}

$\beta$-cells present an extremely difficult imaging target in the clinic, in part because of their low fraction $(\sim 1 \%)$ in the pancreas and the small size of islets, resulting in significant volume averaging with surrounding exocrine tissue. In addition, as Willekens et al. have shown (2), the expression of GLP-1R has significant interspecies variability. The important question for the clinic is if and how much GLP-1R protein expression exists in off-target cells in humans. Specifically, expression of GLP-1R on exocrine cells is critical, since even expression levels 100-fold lower than $\beta$-cells can still be significant given their abundance relative to $\beta$-cells, and estimates have indicated that 1,000-fold lower levels are needed for $\beta$-cell mass quantification (3).

Unfortunately, many studies cited previously (4) indicate measurable expression of GLP1-R protein in human exocrine cells (5). The importance of quantifying target expression cannot be overstated, and this has to be done at the protein level. In this case, the transcriptional data are not representative of protein levels, which are the relevant metric for molecular imaging. For example, the messenger RNA expression data from Willekens et al. show similar mouse and rat messenger RNA endocrine-to-exocrine ratios despite very different protein levels (2). Absolute quantification (i.e., number of receptors per cell) is critically important. The "small differences in exocrine pancreatic uptake between wild-type and GLP-1R knockout mice" on a per-cell basis translate into over half of the total signal in the pancreas because of their 100-fold higher prevalence. For mice, we quantified 54,000 GLP-1R per $\beta$-cell and approximately 50-fold lower levels for exocrine cells ( $\sim 40$-fold lower based on single-cell flow cytometry measurements (4) and 60-fold lower based on bulk percentage injected dose/g (6)). Indeed, 1,000 receptors per cell is below the limit of detection for most single-cell and tissue fluorescent methods and can easily be overlooked compared with the intense $\beta$-cell staining. Despite this difficulty in detection, a robust signal unfortunately remains, and this level in mice is well above the limits necessary to cause problems in whole-body imaging.

\section{RELEVANCE}

To clarify, we do not claim that "the lack of clinical distinction between healthy volunteers and subjects with long-term diabetes" was caused by exocrine uptake, but rather the full sentence indicates this is a possible result consistent with the evidence. To rigorously and unambiguously identify residual $\beta$-cell mass in patients, uptake from the exocrine pancreas has to be discounted.

Even more problematic, Waser and Reubi demonstrated that only about half of human samples (3/5) appear to express detectable exocrine signal (7). If it were uniform among patients, we would agree that some long-standing type 1 diabetic patients showing background levels would support a lack of exocrine uptake. However, since some patients appear to lack exocrine expression, the background levels could simply be the patients lacking exocrine expression. Now in contrast, the preliminary results reported by Gotthardt et al. indicate less variability in the 7 patients they studied. Here, the endocrine-to-exocrine ratio was $3.9 \pm 0.5$, with little variation $(8)$. However, this number is similar to the ratios they reported in mice (4.11 \pm 0.9 and $4.56 \pm 0.9)$ rather than rats (44-106) (2). These clinical data appear consistent with histology reports of significant human exocrine GLP-1R expression $(9,10)$.

The fact that these clinical data are similar to mice does not mean that the absolute expression levels are the same in mice and humans. At the tracer doses used, the similarity is more likely a reflection of delivery, that is, higher vascularization in endocrine tissue than in exocrine tissue. This is why the mouse autoradiography ratio is only approximately 4 at tracer doses whereas the absolute expression differences we measured are close to 50 at saturating doses. It is currently unclear what this ratio is in humans.

\section{WHERE DO WE GO FROM HERE?}

The most recent data reported by Gotthardt and colleagues are exactly the type of data that need to be collected if we want to determine whether the exocrine uptake can be selectively blocked (8). I commend them for their significant efforts to collect these important data, since pancreatic samples are difficult to obtain yet exactly the type of data the scientific community needs. Similar to our collaboration using intraoperative imaging agents to look at antibody distribution in tumors and associated healthy tissue (11), ex vivo analysis can enable absolute probe uptake after in vivo administration.

If their preliminary results continue to mimic the mouse pancreas, selective blocking may be needed to suppress exocrine uptake and reliably detect $\beta$-cells as we propose. The method we used in mice is not perfect, and the absolute endocrine-to-exocrine expression ratio in humans likely needs to be greater than in mice to practically work in humans. However, potential blocking agents are available that could be used in a similar study. Although we used a lipophilic fluorescent dye to simultaneously bind albumin and facilitate imaging, a lipophilic conjugate such as liraglutide would be a potential Food and Drug Administration-approved blocking agent.

The path is difficult, but it is an exciting time to investigate $\beta$-cell biology. Importantly, these results do not discount the potential presence of $\beta$-cells within the diabetic pancreas. Rather, they indicate that the exocrine expression level has to be addressed to definitively image residual $\beta$-cell mass within the human pancreas. The method outlined by Khera et al. (4) is one approach that can be pursued in humans to address this issue. The work by the Gotthardt lab and others staining for $\beta$-cell markers such as GLP-1R in patients with long-standing diabetes raises the possibility of reversing this disease. To interpret these imaging results, we need to definitively know the endocrine-versus-exocrine uptake of these molecular probes. Exendin has many attributes of an ideal imaging agent - tight binding, high retention from metabolic trapping, low nonspecific sticking, and rapid clearance. We just need to ensure the cellular specificity to be able to use this agent in the clinic.

\section{REFERENCES}

1. Gotthardt M, Joosten L, Jansen TJP, Brom M, Boss M, Willekens SMA. From mice to humans: the exocrine pancreas does not matter in human GLP-1 receptor imaging [letter]. J Nucl Med. 2021;62:745.

2. Willekens SM, Joosten L, Boerman OC, et al. Strain differences determine the suitability of animal models for noninvasive in vivo beta cell mass determination with radiolabeled exendin. Mol Imaging Biol. 2016;18:705-714.

3. Sweet IR, Cook DL, Lernmark A, Greenbaum CJ, Krohn KA. Non-invasive imaging of beta cell mass: a quantitative analysis. Diabetes Technol Ther. 2004;6:652-659.

4. Khera E, Zhang L, Roberts S, et al. Blocking of glucagonlike peptide-1 receptors in the exocrine pancreas improves specificity for $\beta$-cells in a mouse model of type 1 diabetes. J Nucl Med. 2019;60:1635-1641.

5. Pyke C, Heller RS, Kirk RK, et al. GLP-1 receptor localization in monkey and human tissue: novel distribution revealed with extensively validated monoclonal antibody. Endocrinology. 2014;155:1280-1290. 
6. Zhang L, Thurber GM. Quantitative impact of plasma clearance and down-regulation on GLP-1 receptor molecular imaging. Mol Imaging Biol. 2016;18:79-89.

7. Waser B, Reubi JC. Radiolabelled GLP-1 receptor antagonist binds to GLP-1 receptor-expressing human tissues. Eur J Nucl Med Mol Imaging. 2014;41:1166-1171.

8. Gotthardt M, Jansen TJP, Buitinga M, et al. Validation of exendin for beta cell imaging: ex vivo autoradiography of human pancreas demonstrates specific accumulation of radiolabeled exendin in islets of Langerhans [abstract]. Diabetologia. 2020;63:393.

9. Körner M, Stockli M, Waser B, Reubi JC. GLP-1 receptor expression in human tumors and human normal tissues: potential for in vivo targeting. J Nucl Med. 2007;48:736-743

10. Kirk RK, Pyke C, von Herrath MG, et al. Immunohistochemical assessment of glucagon-like peptide 1 receptor (GLP-1R) expression in the pancreas of patients with type 2 diabetes. Diabetes Obes Metab. 2017;19:705-712.

11. Lu G, Nishio N, van den Berg NS, et al. Co-administered antibody improves penetration of antibody-dye conjugate into human cancers with implications for antibody-drug conjugates. Nat Commun. 2020;11:5667.

Greg M. Thurber

University of Michigan

Ann Arbor, MI

E-mail: gthurber@umich.edu

Published online Mar. 6, 2021.

DOI: 10.2967/jnumed.121.262029

\section{Disparities in PET Imaging of Prostate Cancer at a Tertiary Academic Medical Center}

TO THE EDITOR: We read with interest the article by Bucknor et al. titled, "Disparities in PET Imaging for Prostate Cancer at a Tertiary Academic Medical Center" (1). The authors compare enrollment data between 2 cohorts: one having standard-of-care (SOC) ${ }^{18} \mathrm{~F}$-fluciclovine PET and a second undergoing ${ }^{68} \mathrm{Ga}$-prostate-specific membrane antigen (PSMA)-11 PET. As SOC, ${ }^{18} \mathrm{~F}-$ fluciclovine PET is generally eligible for reimbursement by insurance whereas ${ }^{68}$ Ga-PSMA-11 PET was offered under a Food and Drug Administration-reviewed investigational new drug protocol with cost-recovery mechanism.

More participants were reported in the investigational arm $(1,502,85.5 \%)$ than in the SOC arm $(254,14.5 \%)$ over the same period. The authors indicate that the proportion of African Americans who had SOC PET was $6.7 \%$, as opposed to a mere $1.4 \%$ in the investigational arm. The percentages for Asians were $8.7 \%$ and $5.8 \%$, respectively, and for Whites, $71.6 \%$ and $80 \%$, respectively. The 2010 San Francisco Bay Area Census indicates a population distribution of $6.7 \%$ African Americans, 23.3\% Asians, and $52.5 \%$ Whites. Although the proportion who had SOC PET was aligned with the geographic racial mix, for the investigational arm the African American inclusion was more than 4 times lower. Finding that African American patients had increased odds of receiving imaging with ${ }^{18} \mathrm{~F}$-fluciclovine versus ${ }^{68} \mathrm{Ga}-\mathrm{PSMA}-11$, compared with non-Hispanic White patients, the authors conclude that access to ${ }^{68} \mathrm{Ga}$-PSMA-11 for African American patients was limited, compared with White patients.

The authors acknowledge the limitations of a single-site study. As a point of reference, our institution is located in the same geographic area (Northern California). We started a second program for PSMA PET imaging in May 2018 using 2-(3-\{1-carboxy-5[(6- ${ }^{18}$ F-fluoro-pyridine-3-carbonyl)-amino]-pentyl $\}$-ureido)-pentanedioic acid $\left({ }^{18} \mathrm{~F}-\mathrm{DCFPyL}\right)$ at biochemical recurrence of prostate cancer (NCT03501940) (2), after completing a phase II study of ${ }^{68}$ Ga-PSMA-11 (NCT02673151). In total, 187 participants have been enrolled to date in the investigational cohort, whereas 436 patients have undergone SOC ${ }^{18} \mathrm{~F}$-fluciclovine PET over the same period. The proportion of African Americans who had SOC PET was $4.4 \%$, versus $4.8 \%$ in the investigational arm. The respective percentages were $13.1 \%$ and $8.6 \%$ for Asians and $68 \%$ and $79.7 \%$ for Whites.

How can 2 institutions be so geographically close yet have such a different experience in equitable access to care through a research trial? Part of the answer may be related to the need to include a more complete set of predictor variables. For example, the amount of the health-care expenditure for which the patient is held responsible, rather than merely the classification of insurance as "commercial," "government," or "unknown," may be more telling of a patient's ability to pay in an era of significant copayments and high deductibles (3). In addition, the authors, as well as other contributors to the literature (4), point out several other patient-specific factors that could be considered as predictors in future studies. However, referral to a tertiary- or quaternary-care center for imaging may have more to do with the behavior of the referring provider or the number of physicians involved in the care of the patient than with characteristics of the patients themselves (5).

The different results between our geographically close institutions may also be a direct result of inadvertent effects of trial design. The authors state, "Remarkably, despite the requirement for study participation and the possibility of self-pay, nearly six times as many patients in this study were imaged with ${ }^{68}$ Ga-PSMA-11 compared to ${ }^{18}$ F-fluciclovine," and go on to highlight potential disparities in access to imaging research trials for African-American patients. The Food and Drug Administration-approved costrecovery mechanism used to pay for ${ }^{68} \mathrm{Ga}$-PSMA-11 in the study allows institutions to charge private insurance (not Medicare) and individuals the direct cost of manufacturing the radiopharmaceutical, audited by an external certified public accountant. However, the cost-recovery mechanism does not govern the charges for technical and professional fees for a PET/CT examination. Bucknor et al. indicate a charge associated with cost recovery at their institution ranging between $\$ 900$ and $\$ 1,400$, depending on the number of syntheses performed in a year; however, although mentioned briefly, they do not detail the technical and professional fees billed to participants or insurance in their protocols. We expect these fees to be at least as much as cost recovery for the radiopharmaceutical dose, based on known Medicare charges.

At our institution, we applied for a research access program through the Prostate Cancer Foundation in 2017. On approval, ${ }^{18} \mathrm{~F}$-DCFPyL was provided at no cost and we waived the technical and professional fees for all participants. Therefore, the participants who have PSMA PET at our institution do not receive bills related to the radiopharmaceutical, imaging acquisition, or report.

Although very important to bring novel radiopharmaceuticals to the United States, cost-recovery trials may create unequal access when there are no mechanisms to provide the same opportunities for disadvantaged patient groups. As the authors themselves point out, "Through this mechanism, patients often would be financially liable for the direct cost of the radiotracer and possibly the cost of the technical component of the PET imaging, which could pose a significant barrier to low income groups." Barriers to care access can result from bias (perceived or unperceived), shortcomings of research recruitment strategies, or geographic availability of services. However, barriers can also be created by the threat of 